\title{
Permissible parameters for the circulation rate of the sludge mixture in airlift reactor-clarifier with suspended layer
}

\author{
Viktor Nezdoiminov ${ }^{1}$, Dmitrii Zavorotnyi ${ }^{1 *}$, Vitalii Rozhkov ${ }^{1}$, and Pavel Deminov ${ }^{1}$ \\ ${ }^{1}$ Donbas National Academy of Civil Engineering and Architecture, Faculty of Engineering and \\ Environmental Systems, Derzhavin str. 2, Makeevka, Donetsk People's Republic
}

\begin{abstract}
Factors affecting the permissible limits of sludge mixture circulation rate are given for airlift reactor-clarifier with a suspended layer. The upper limit of the circulation rate is justified as a function of some geometric dimensions of the bioreactor and the hydraulic load on the clarifier to comply with conditions of a suspended layer existence. The lower limit of the circulation rate was obtained on the basis of two conditions: the duration of the active sludge stay in anoxic conditions and the observance of recommended rates in the construction in order to avoid the settling of active sludge. Theoretical and empirical dependences of the maximum permissible values of the circulation rate on the main geometric parameters and on productivity of the airlift reactor-clarifier are presented.
\end{abstract}

\section{Introduction}

In recent years, airlift reactors are widely used for a variety of biological and chemical processes [1-3]. In particular, these facilities may contain activated sludge and serve for aerobic wastewater treatment $[4,5]$. The aeration system of airlift bioreactors with internal circulation is a flooded airlift, i.e. airlift, the upper section of which is located below the free surface of the aerated fluid. Such facilities allow combining the processes of nitrification and denitrification, which positively affects the technical and economic efficiency of wastewater treatment from nitrogen compounds and organic pollutants [6-9]. In addition to biochemical treatment, biological treatment facilities serve for clarifying the liquid from activated sludge [10].

The Donbas National Academy of Civil Engineering and Architecture has developed a biological treatment facility in which an airlift bioreactor with internal circulation is combined with a clarifier i.e. airlift reactor-clarifier with a suspended layer (fig. 1) [11]. In this construction, clarification is carried out by means of a being formed suspended layer of activated sludge.

In comparison with the most existing bioreactors combined with clarifiers are used in domestic sewage treatment, the airlift reactor-clarifier with a suspended layer has the following advantages:

\footnotetext{
${ }^{*}$ Corresponding author: d.v.zavorotnyi@ donnasa.ru
} 
i sludge movement from the suspended layer to the aerated part is carried out by means of hydraulic sludge recirculation, without additional equipment and formation of active sludge precipitation zones;

ii a high proportion of the total volume is filled with a sludge mixture and, accordingly, takes part in wastewater treatment.

a

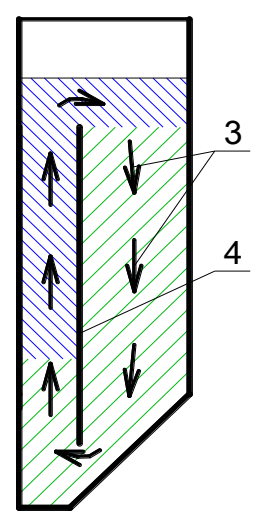

b

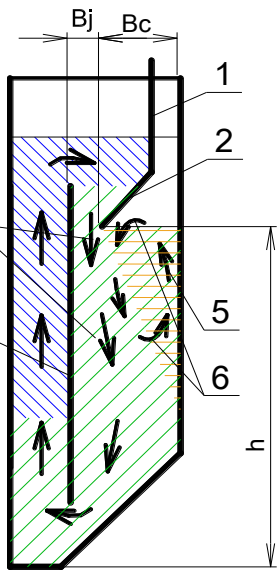

- zone of saturation of the sludge mixture with atmospheric oxygen

- oxygen consumption zone

- zone of activated sludge suspended layer formation

Figure 1. Schematic drawing of (a) the airlift bioreactor with internal recirculation and (b) the airlift reactor-clarifier with a suspended layer.

The airlift reactor-clarifier with suspended layer contains a partition 1 with an inclined peak 2, which separate the clarified liquid from the sludge mixture. When the downward flow 3 passes between the inclined peak and the wall of the flooded air-lift 4, it undergoes compression. As a result of compression, the downward flow ejects the sludge mixture from adjacent layers of liquid and its flow rate increases. Some part of the downward flow returns to the flooded airlift through the gap between its base and the bottom of the tank. The other part of the downward flow changes direction and forms an ascending stream 5 near the outer wall of the airlift reactor-clarifier. Thus, under the peak a vortex zone 6 is formed.

The upward flow near the outer wall of the reactor-clarifier maintains the sludge in a suspended state. At the level of the lower edge of the inclined peak, the surface of the suspended layer is set up. At this level, the sludge mixture in the suspended layer moves horizontally in the direction of the downward flow. The vortex zone is located in the area behind the streamlined body (peak). Vortex formation occurs at the boundary between the sludge mixture, which circulates through the flooded airlift and the vortex zone [12]. Vortex formation at the boundary of two flows contribute to the mutual flow of particles between the volumes of the vortex zone and the circulation flow of the airlift reactor.

The facilities of wastewater biological treatment must adhere to the hydrodynamic conditions [13, 14]. The parameters of the liquid circulation in the airlift reactor-clarifier with suspended layer can be limited in terms of maintaining a number of technological processes: mixing the activated sludge in the biological reactor, providing conditions for filtering the sludge mixture in the suspended layer, observing the residence time of the biochemically purified liquid in the corresponding zone of the reactor, etc.

The analysis of the criteria for determining permissible circulation was not covered in the literature.

\section{Methods}

The circulation intensity is taken as the main parameter characterizing the circulation rate of the bioreactor flow: 


$$
\mathrm{I}_{\mathrm{c}}=\mathrm{Q}_{\mathrm{a} . \mathrm{h}} / \omega_{\mathrm{j}},\left[\mathrm{L} \cdot \mathrm{T}^{-1}\right],
$$

where $\mathrm{Q}_{\mathrm{a}, \mathrm{h}}$ is the hour fluid flow in the gap between the wall of the flooded airlift and the lower edge of the inclined peak, $\left[\mathrm{L}^{3} \cdot \mathrm{T}^{-1}\right]$.

$\omega_{\mathrm{j}}$ is the sectional area of the gap between the wall of the flooded airlift and the lower edge of the inclined peak, $\left[\mathrm{L}^{2}\right]$.

The dependence of the maximum permissible values of the circulation intensity $\mathrm{I}_{\mathrm{c}, \mathrm{s}}$ on the hydraulic load on the suspended layer of bioreactor obtained experimentally. $\mathrm{I}_{\mathrm{c}, \mathrm{s}}$ was defined as the maximum value of the circulation intensity at which the increase in the level of the suspended layer does not lead to its smearing and removal of the sludge flakes with clarified liquid [11]. The possibility of application of relations which were obtained in vitro, for calculating scale of the production facilities may be confirmed by simulation using criteria of Reynolds and Froude [15].

Long-term presence of active sludge in anoxic conditions can adversely affect its physiological characteristics. The downward flow, passing through the gap between the peak and the wall of the flooded air-lift, is a flat flooded wall turbulent jet. Calculation of the circulation intensity, which provides a sufficient update of the sludge mixture in anoxic zone, is based on the relations which describe the velocity distribution in flooded turbulent jets [16, 17] and in the turbulent wall-adjacent layer [18]. The actual residence time of the sludge mixture in the suspended layer is calculated by the formula 2 :

$$
t=\frac{h \cdot\left[B_{c}-h \cdot \operatorname{tg}\left(\alpha_{2}\right) / 2\right]}{Q_{w}-Q_{s} \cdot S / h},
$$

where $\mathrm{B}_{\mathrm{c}}$ is the distance from the lower edge of the inclined peak to the outer vertical wall of the bioreactor, [L] (Fig. 1);

$\mathrm{h}$ is the distance from the bottom edge of the inclined peak to the bottom of bioreactor, [L]; [16];

$\alpha_{2}$ is the the angle between the vertical and the outer boundary of the outer part of the jet

$\mathrm{Q}_{\mathrm{s}}$ is the productivity of bioreactor, $\left[\mathrm{L}^{3} \cdot \mathrm{T}^{-1}\right]$;

$\mathrm{S}$ is the depth of the conditional center of the vortex zone relative to the lower edge of the inclined peak, [L];

$\mathrm{Q}_{\mathrm{w}}$ is the fluid flow rate rising in the suspended layer at a depth $\mathrm{S}$ relative to the lower edge of the inclined peak, which should be calculated without taking into account the effect of the hydraulic load:

$$
Q_{w}=\left[B_{c}-S \cdot \operatorname{tg}\left(\alpha_{2}\right)\right] \cdot \frac{L \cdot I_{c} \cdot S \cdot\left[0,135-\operatorname{tg}\left(\alpha_{1}\right)-0,125 \cdot \delta_{0} / S_{0}\right]}{3600 \cdot\left\{B_{c}+S \cdot\left[0,165-\operatorname{tg}\left(\alpha_{2}\right)\right]\right\}},
$$

where $\alpha_{1}$ is the the angle between the vertical and the inner boundary of the outer part of the jet [16];

$\mathrm{S}_{0}$ is the length of the initial section of a flooded turbulent stream, [L] [16];

$\delta_{0}$ is the width of the wall portion of the jet at a distance $S_{0}$ of the lower edge of the inclined peak, $[\mathrm{L}]$;

$\mathrm{L}$ is the width of a rectangular bioreactor, [L].

The minimum permissible circulation intensity $\mathrm{I}_{\mathrm{c}, \mathrm{t}}$ is the minimum value of the circulation intensity $I_{c}$ in formula 3 , at which time $t$ in formula 2 does not exceed the permissible time of the sludge residence under anoxic conditions.

To prevent bottom deposits, the intensity of circulation should ensure the existence of appropriate velocities at the bottom of the bioreacror. The velocities that prevent siltation of the reactor bottom are determined by the empirical formulas presented in the engineering literature [19]. Taking into account the dependence of the velocity at the bottom on the circulation intensity from the theory of turbulent jets: 


$$
I_{c, v}=15900 \cdot v_{s} \cdot H^{0,22} \cdot \sqrt{\frac{0,24 \cdot h}{B_{j}}+0,41},
$$

where $I_{c, v}$ is the minimum allowable circulation rate at which the flow velocities at the bottom of the bioreactor prevent the formation of deposits, $\left[\mathrm{L} \cdot \mathrm{T}^{-1}\right]$;

$\mathrm{v}_{\mathrm{s}}$ is the sedimentation rate of the sludge flakes, which depends on the properties of the activated sludge and is about $3 \cdot 10^{-3} \mathrm{~m} / \mathrm{s}[19,20]$;

$\mathrm{B}_{\mathrm{j}}$ is the width of the gap between the wall of the flooded airlift and the lower edge of the inclined peak, [L];

$\mathrm{H}$ is the operational height of airlift reactor-clarifier, [L].

The intensity of circulation maintained in the airlift reactor-clarifier with a suspended layer should be no more than $I_{c, s}$ and not less than $I_{c, t}$ and $I_{c, v}$.

\section{Results and Discussion}

The empirical formula 5 was obtained by processing experiment data to determine maximum values of circulation rates at which no washing out of suspended layer occurs.

$$
I_{c, s}=\left(\frac{74,2}{q+0,29}\right)^{0,801} \cdot\left(\frac{h}{B_{j}}\right)^{0,844},
$$

where $\mathrm{I}_{\mathrm{c}, \mathrm{s}}$ is the maximum allowable circulation rate at which smearing of the suspended layer is prevented, $\left[\mathrm{L} \cdot \mathrm{T}^{-1}\right]$;

$\mathrm{q}$ is the calculated hydraulic load, $\left[\mathrm{L} \cdot \mathrm{T}^{-1}\right]$, which is calculated by formula 6 .

$$
\mathrm{q}=\mathrm{Q}_{\mathrm{s} . \mathrm{h}} /\left(\mathrm{B}_{\mathrm{c}} \cdot \mathrm{L}\right)
$$

where $\mathrm{Q}_{\mathrm{s}, \mathrm{h}}$ is the hourly output of airlift reactor-clarifier, $\left[\mathrm{L} \cdot \mathrm{T}^{-1}\right]$;

Figure 2, 3 show the graphs of the limiting permissible values of the circulation intensity $\mathrm{I}_{\mathrm{c}}$. The permissible circulation intensity, mainly, depends on the hydraulic load $\mathrm{q}$ and the geometric parameters of the airlift reactor-clarifier.

Practically, high productivity and small dimensions are preferable for any facility. The value of the hydraulic load in the airlift reactor-clarifier with suspended layer is limited. At high values of the circulation rate, the hydraulic load is limited in order to prevent washing out of the suspended layer. At low circulation rates, high hydraulic loads can prevent recirculation of sludge from the suspended layer to the aerated part of the structure. Relatively low values of hydraulic load will allow to vary the intensity of circulation in a wide range.

The height of the airlift reactor-clarifier with a suspended layer is of great importance in determining the permissible hydrodynamic parameters. Theoretical data indicate that an insufficient height of the suspended layer makes it very difficult to return the sludge from the suspended layer. 


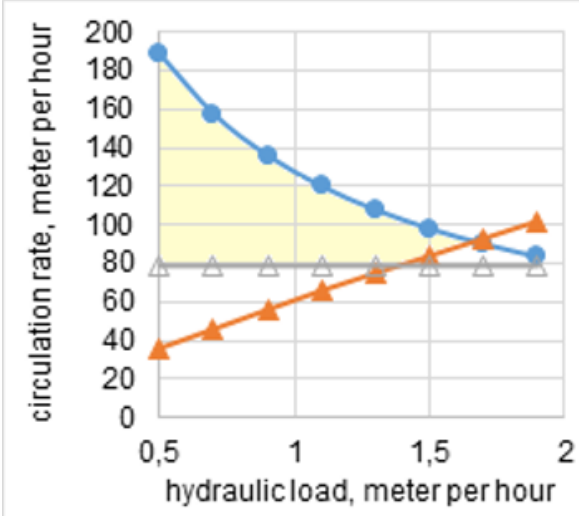

\section{$\longrightarrow$ maximumvalues of circulation} rates Ic, s

- minimumvalues of circulation rates Ic, t,

$\_$minimum values of circulation rates Ic, $v$

Figure 2. Limits of the permissible values of the circulation rate depending on the hydraulic load at the width of the gap $\mathrm{Bj}=0.3 \mathrm{~m}$ and the height of the inclined peak relative to the bottom of the airlift reactor-clarifier $\mathrm{h}=2 \mathrm{~m}$.

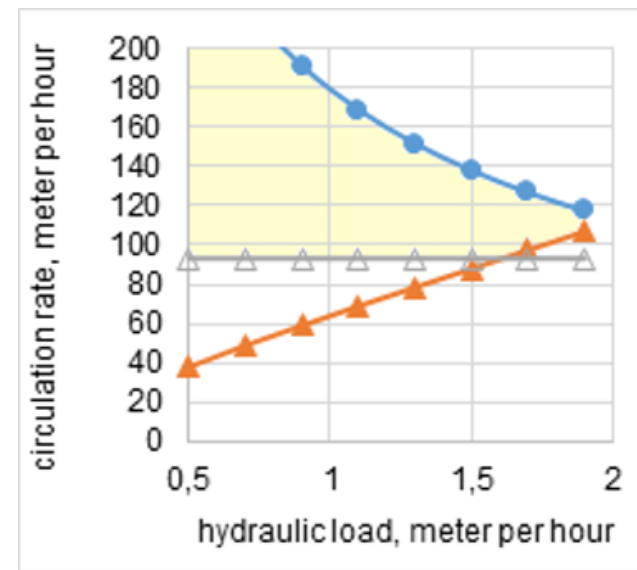

(a)

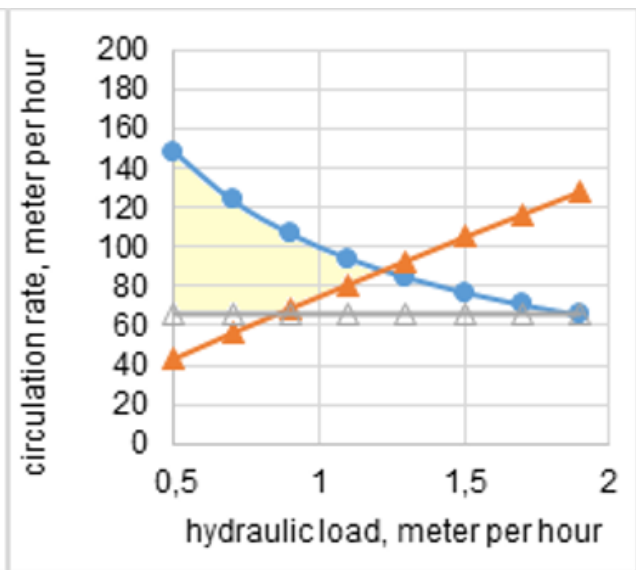

(b)

Figure 3. Limits of the permissible values of the circulation rate depending on the hydraulic load (a) at the width of the gap $\mathrm{Bj}=0,2 \mathrm{~m}$ and the height of the inclined peak relative to the bottom of the airlift reactor-clarifier $h=2 \mathrm{~m},(\mathrm{~b})$ at the width of the gap $\mathrm{Bj}=0,3 \mathrm{~m}$ and the height of the inclined peak relative to the bottom of the airlift reactor-clarifier $\mathrm{h}=1,5 \mathrm{~m}$.

\section{Conclusions}

The criteria for determining the minimum permissible circulation intensity in the airlift reactor-clarifier with suspended layer are analyzed. The operation areas of facilities in the field of hydraulic loading and the intensity of circulation are presented.

1. Prevention of siltation is facilitated by high values of the width of the gap between the inclined peak and the wall of the flooded air-lift, as well as low values of the height of the peak. However, from the point of view of preventing silt, the main geometric parameters have little effect on the minimum allowable circulation rate.

2. Dependencies are presented for calculation of the minimum allowable circulation rate to maintain sufficient recirculation of the sludge mixture. The value of the minimum permissible circulation intensity heightens with an increase in the hydraulic load and with a decrease in the depth of the airlift reactor-clarifier relative to the bottom of the peak. 


\section{References}

1. M. Al-Mashhadani, S. Wilkinson, W. Zimmerman, Chem. Engineering Science, - 137, Pp. 243-253. (2015).

2. H.Ghasemi, S.Hosseini, Brazil. Journal of Chem. Engineering, 29 (04), 821-833 (2012).

3. T.Kent, C.Bott, Z-W.Wang, Biotech. Advances, 36 (4), 1139-1166 (2018).

4. P.Cozma, M.Gavrilescu, Env. Engineering and Management Journal, 11 (8), 1505-1515 (2012).

5. V.Nezdoiminov, Vestnik Donb. nation. Akademii Stroitelstva i Arhitektury, 5, 73-77 (2014).

6. P.Wunderlin, J.Mohn, A.Joss, L.Emmenegger, H.Siegrist, Wat. Res., 46, 1027-1037 (2012).

7. V.Nezdoiminov, V.Ziatina, V.Rozhkov, D.Nemova, Proc. Engineering, 117, 1022-1027 (2015).

8. M.Kim, J. DeFrank, J.Lee, H.Kim, Water-Energy Nexus, 1, 47-55 (2018).

9. Y.Krishna Reddy, S.Adamala, E.Levlin, K.Reddy, Int. Journal of Sustainable Built Environment, 6, 351-358 (2017).

10. Z.Bakiri, D.Chelli, S.Nasef, Energy Procedia. 18, 1-9 (2012).

11. D.Zavorotnyi, Sbornik nauchnyh trudov GOU VPO LNR DonGTU, 10, 104-109 (2018).

12. A.Kalyakin, E.Chesnokova, Magazine of Civ. Engineering, 3, 49-56 (2015).

13. A.Karpinska, J.Bridgeman, Wat. Res., 88, 861-879 (2016).

14. M.Meister, D.Winkler, M.Rezavand, W.Rauch, Comp. \& Chem. Engineering, 99, 1-12 (2017).

15. I.Kantardgi, M.Zheleznyak, Magazine of Civ. Engineering, 6, 49-59 (2016).

16. G.Abramovich, Teoria turbulennyh Struy, 715 p. (1960).

17. V.Tupov, A.Taratorin, Proc. Engineering, 176, 194-198 (2017).

18. I.Rylova, Magazine of Civ. Engineering, 3, 40-52 (2016).

19. L.Braginskiy et al. Modelirovanie aeracionnyh sooruzheniy, 144 p. (1980).

20. M. Hribersek, B. Zajdela, A.Hribernik, M.Zadravec, Wat. Res., 45, 1729-1735 (2011). 